\title{
Aberrant peripheral nerve fibres in the medulla oblongata of man
}

\author{
WILLIAM DEMYER ${ }^{1}$ \\ From Indiana University School of Medicine, Indianapolis, Indiana, U.S.A.
}

Occasionally peripheral axons with Schwann cell sheaths penetrate the neuraxis. In many instances, the fibres are misdirected nerve rootlets regenerating in proximity to an intra-axial lesion such as traumatic cysts, inflammation, or neoplasm (Klintworth, 1964; Druckman and Mair, 1953; Hughes and Brownell, 1963). In other instances (Staemmler, 1939; Riggs and Clary, 1957), as in the present example of aberrant fibres within the medulla oblongata, no local intra-axial lesion was apparent.

\section{MATERIAL AND METHODS}

The patient, an 82-year-old white man, died with emphysema and renal failure. He had been blind for several years because of cataracts. On admission, he was semistuporous. His chart recorded no other neurological deficits, either by history or physical examination, but he had not been interrogated or examined by a neurologist. He had no signs of neurofibromatosis clinically or on necropsy. The cerebral vessels were sclerotic. The left occipital lobe was infarcted, and the brain-stem, basal ganglia, and the right cerebellar hemisphere showed a few small, scattered ischaemic areas. Grossly the medulla oblongata was normal.

The patient was one of 14 consecutively necropsied, from whom medullae were selected for the purpose of studying the junction zone of the area postrema with the funiculus separans (DeMyer, 1965, in preparation). After fixation by immersion in $4 \%$ formaldehyde, the medullae were embedded in paraffin. Serially cut at $16 \mu$, the sections were alternately stained by Nissl and Spielmeyer methods, and silver impregnated after Hortega for neurites, as adopted for paraffin sections (DeMyer, 1958). The sections were continued caudally through the obex to the level of the central canal of the spinal cord.

\section{OBSERVATIONS}

Of the 14 serially sectioned medullae oblongata, only the 82-year-old man's had peripheral nerve fibres within the neuraxis. Aberrant fibres first appeared in the caudal rootlets of the vagus nerve

'Special fellow, National Institute of Neurological Diseases and Blindness, United States Public Health Service (NB 18404) and were distributed bilaterally, as shown in Figure 1. Some penetrated the lateral aspect of the medulla along the usual course of the vagal rootlets, as shown at point 1 in Figure 1. Others could be traced dorsally outside of the pia mater, around the circumference of the medulla. Some fibre bundles entered the neuraxis in company with blood vessels, particularly with the long circumferential vessels penetrating dorsolateral to the nucleus solitarius (point 2 of Figure 1). The most dorsal site of penetration was just lateral to the lingula (point 3 of Figure 1). At point 3 , the bundles of axons directly penetrated the pia mater and mingled with the subpial glia. Once inside the neuraxis, the fibres passed through the area postrema, extending along vessels as far ventral as the hypoglossal nucleus. From the sites of pial perforation, the number of axons dwindled in proportion to their distance from the surface. Identification of the aberrant fibres, whether intraaxial or extra-axial, was easy because the axons had a very tangled, interlacing course as shown in Figure 2.

Typical Schwann cells accompanied the axons intra- and extra-axially. The calibre of the axons

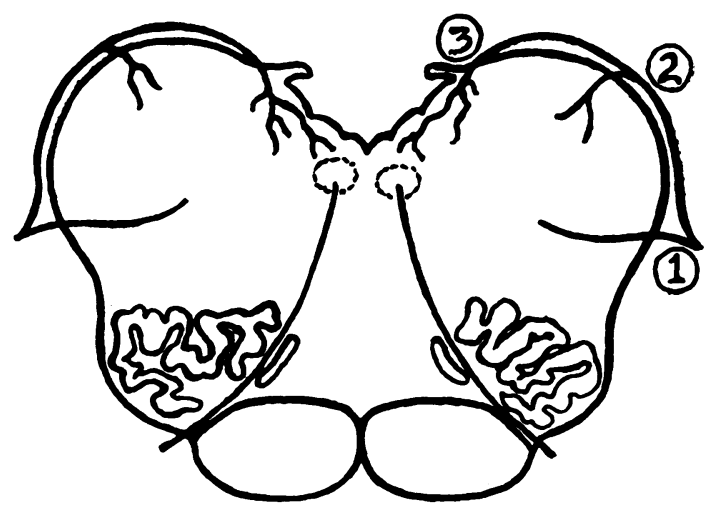

FIG. 1. The dark lines encircling the dorsal aspect of the medulla oblongata illustrate the bilateral course of the aberrant peripheral nerve fibres. The numbers show the sites of penetration of the neuraxis. 


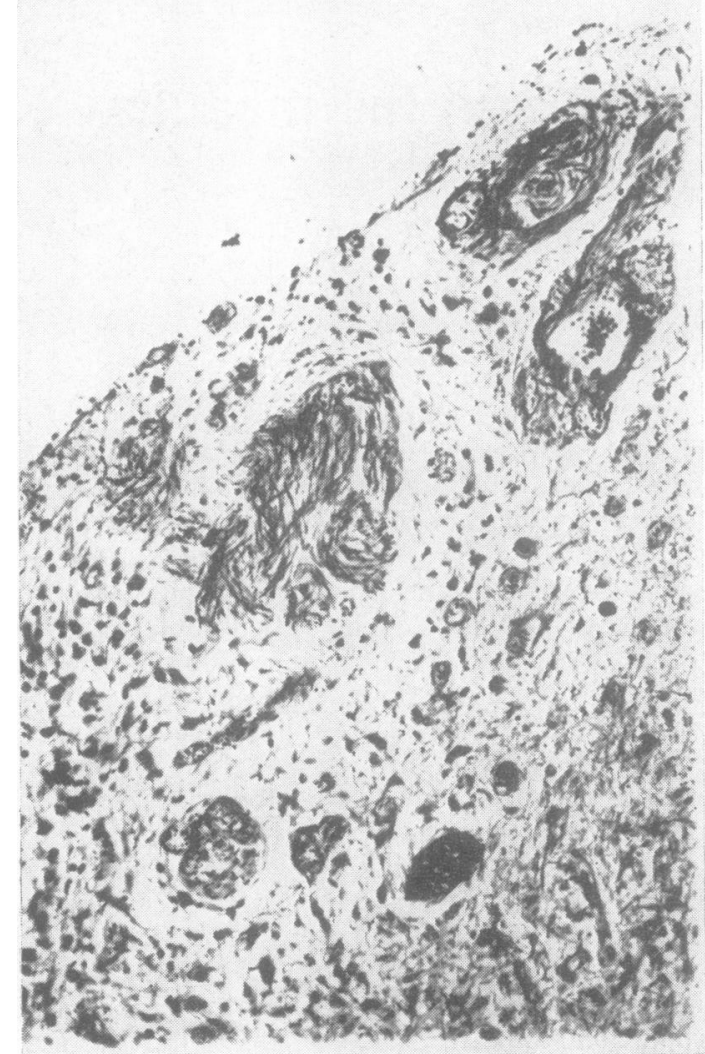

FIG. 2. Silver carbonate impregnation which shows peripheral nerve fibres coursing through the area postrema. The ventricular surface of the area postrema is on the upper left. At the upper right, the nerve fibre bundles are seen around large vessels. In the middle of the field, the large fibre bundle is accompanied only by a capillary. Notice the characteristic whorled, interlacing course of the axons.

varied from small to large. Many of them were in the $10-15 \mu$ range. In the Spielmeyer stain, only a few of the intra-axial peripheral nerve fibres had myelin sheaths, but when extra-axial, the fibres were well myelinated.

Collagen fibres, corresponding to endo- and perineurium, could be found between and around the axons throughout their course. Collagen fibres were identified by their typical appearance in silverimpregnated sections. When recently-fixed material is impregnated with silver carbonate, axons are deep purple to black, while collagen is a bright pink. Tinctorial as well as morphological features permit a fairly reliable distinction to be made.

Although the medulla of this patient showed scattered axonal degeneration and other changes compatible with senility, neither the parenchyma of the medulla nor the meninges showed any lesions, such as inflammation or infarction, which might have predisposed to the ingrowth of axons. However, the vessels were sclerotic, and the right cerebellar hemisphere had small ischaemic areas.

\section{DISCUSSION}

Any doubt that the structures identified as nerves contain axons is dispelled immediately by the completely characteristic appearance of the axons in the silver carbonate impregnation and by their continuity with rootlets of the vagus nerve. The point of major interest is the pathogenesis of the aberrant fibres. Most likely, they arose from dorsal root ganglia or ventral root fibres of the vagus nerve. The well-reasoned arguments of previous authors against interpreting intra-axial peripheral nerve bundles as normal perivascular fibres need not be repeated here. However, the bundles may represent hypertrophic perivascular fibres (Klintworth, 1964; Riggs and Clary, 1957; Lu, Kypridakis, Abbott, and i Vogel, 1963; Pitts and Riggs, 1964). Most author\$ i draw an analogy between the tangled course of introf $\vec{N}$ axial peripheral fibres with amputation neuromas i尺 transected nerves (Klintworth, 1964; Druckman and 응 Mair, 1953, Hughes and Brownell, 1963). The implication is that the cause of the aberrant fibres $\frac{D}{0}$ in the present case would be a transecting lesion followed by regeneration. For many previous cases $\overrightarrow{8}$ the postulated mechanism of transection-regenera: of tion seems amply supported by the presence of an obvious predisposing lesion. In the present case, no lesion was apparent (or scarcely can be imagined) which could account either for the symmetrical disposition of the aberrant fibres or their penetration $\frac{0}{8}$ of an apparently normal pia mater and neuraxis. Inasmuch as previous authors (Riggs and Clary, $\overrightarrow{\overrightarrow{0}}$ 1957; Lu et al., 1963; Pitts and Riggs, 1964) indi- 3 cate that the incidence or at least the prominence of intra-axial peripheral nerve fibres increases with age, one must admit the possibility that the fibres developed by transection-regeneration or some hypertrophic mechanism in later life. The one plausible $\dot{0}$ transecting agent apparent in the current case would $\stackrel{3}{\circ}$ be ischaemic damage to the vagus rootlets from $\delta$ vascular disease, which was prominent.

Experimental studies (Jakoby, Turbes, and Free- $ᄋ$ man, 1960; Turbes and Freeman, 1958) as well as $D$ observations on human material (Freeman, 1961) 을 indicate that regenerating nerve fibres, even after dorsal rhizotomy (Hughes and Brownell, 1963), (r usually fail to penetrate normal meninges, normal $N$ neuraxial substance, or glial scars, although invasion $N$ can be promoted pharmacologically (Windle, 
Clemente, and Chambers, 1952). Apparently an important condition for ingrowth of peripheral axons in postnatal animals is that normal barriers be destroyed or bypassed. Since the fibres in the present case were bilateral, penetrated normal neuraxis and meninges, and intermingled with glia at their penetration points, the question arises whether they grew in during embryogenesis. If so, the one normal element missing at the neuraxial junction was the Obersteiner-Redlich zone. The free ingrowth of Schwann cells with aberrant fibres suggests that the ability of the neuraxis to produce the ObersteinerRedlich zone is very limited temporally or topographically during embryogenesis. If the neuraxis is to construct this glial-Schwann cell barrier, the axons apparently must grow in at the right time and right place during embryogenesis. If one were to assume that the fibres in the current case arose during embryogenesis and not from a post-natal mechanism, the axons would have been wrong in their timing in reaching the vagal rootlet junction zone and wrong in their place in reaching the ligula. Otherwise the Obersteiner-Redlich zone would have formed.

\section{SUMMARY}

In 14 serially sectioned human medullae oblongata, one had peripheral nerve fibres penetrating the neuraxis. Unique features of this case are bilateral symmetry of the fibres, their poor myelination intraaxially, their large, coarse axons, and the apparent absence of a predisposing lesion of the neuraxial wall. The peripheral axons traversed the neuraxial tissue in company with Schwann cell sheaths and blood vessels. They proceeded ventrally as far as the hypoglossal nucleus. Dwindling in number as they went, the axons vanished without their mode of termination being apparent. Their origin, whether during pre-natal or post-natal life, was not clear.

\section{REFERENCES}

DeMyer, W. (1958). Impregnation of axons and terminal buttons in routine paraffin, or frozen sections of central and peripheral nervous tissue. Amer. J. clin. Path., 29, 449-454.

- (1965). The area postrema, area juxtapostrema, and the funiculus separans; comparative anatomy from rat to man. (In preparation).

Druckman, R., and Mair, W. G. P. (1953). Aberrant regenerating nerve fibres in injury to the spinal cord. Brain, 76, 448-454.

Freeman, L. W. (1961). Neuronal regeneration in the central nervous system of man. J. Neurosurg., 18, 417-422.

Hughes, J. T., and Brownell, B. (1963). Aberrant nerve fibres within the spinal cord. J. Neurol. Neurosurg. Psychiat., 26, 528-534.

Jakoby, R. K., Turbes, C. C., and Freeman, L. W. (1960). The problem of neuronal regeneration in the central nervous system. I. The insertion of centrally connected peripheral nerve stumps into the spinal cord. J. Neurosurg., 17, 385-393.

Klintworth, G. K. (1964). Axon regeneration in the human spinal cord with formation of neuromata. J. Neuropath. exp. Neurol., 23. 127-134.

Lu, A., Kypridakis, G., Abbott, K., and Vogel, P. (1963). Intramedullary neurofibromas of the cervical cord. Report of two cases. Bull. Los Angeles neurol. Soc., 28, 31-36.

Pitts, F. W., and Riggs, H. E. (1964). Intramedullary neuromas of the spinal cord. J. Neuropath. exp. Neurol., 23, 200-202.

Riggs, H. E., and Clary, W. U. (1957). A case of intramedullary sheath cell tumor of the spinal cord. Consideration of vascular nerves as a source of origin. Ibid., 16, 332-336.

Staemmler, M. (1939). Beiträge zur normalen und pathologischen Anatomie des Rückenmarks. III. Diffuse Angioneuromatose des Lendenmarks und seiner Pia. Z. ges. Neurol. Psychiat., 166, 529-536.

Turbes, C. C., and Freeman, L. W. (1958). Peripheral nerve-spinal cord anastomosis for experimental cord transection. Neurology (Minneap.), 8, 857-861.

Windle, W. F., Clemente, C. D., and Chambers, W. W. (1952). Inhibition of formation of a glial barrier as a means of permitting a peripheral nerve to grow into the brain. J. comp. Neurol., 96, 359-369. 